\title{
Permanent Spinal Nerve Paralysis
}

National Cancer Institute

\section{Source}

National Cancer Institute. Permanent Spinal Nerve Paralysis. NCI Thesaurus. Code C27316.

Spinal nerve paralysis that is irreversible. 